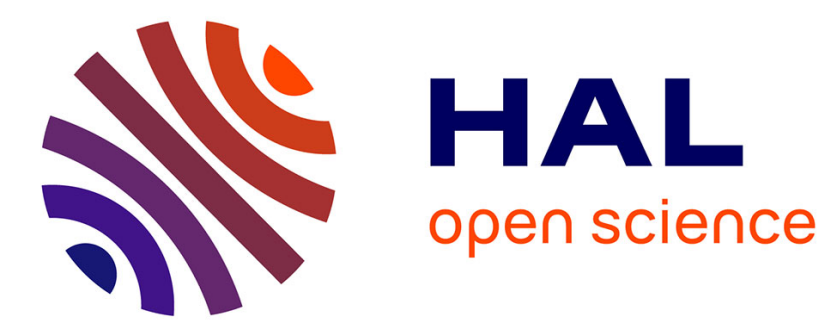

\title{
Computing Minimal Projection Modules for ELHr -Terminologies
}

Jieying Chen, Michel Ludwig, Yue Ma, Dirk Walther

\section{To cite this version:}

Jieying Chen, Michel Ludwig, Yue Ma, Dirk Walther. Computing Minimal Projection Modules for ELHr -Terminologies. European Conference on Logics in Artificial Intelligence, May 2019, Rende, Italy. pp.355-370, 10.1007/978-3-030-19570-0_23 . hal-02930238

\section{HAL Id: hal-02930238 \\ https://hal.science/hal-02930238}

Submitted on 4 Sep 2020

HAL is a multi-disciplinary open access archive for the deposit and dissemination of scientific research documents, whether they are published or not. The documents may come from teaching and research institutions in France or abroad, or from public or private research centers.
L'archive ouverte pluridisciplinaire HAL, est destinée au dépôt et à la diffusion de documents scientifiques de niveau recherche, publiés ou non, émanant des établissements d'enseignement et de recherche français ou étrangers, des laboratoires publics ou privés. 


\title{
Computing Minimal Projection Modules for $\mathcal{E} \mathcal{L} \mathcal{H}^{r}$-Terminologies ${ }^{\star}$
}

\author{
Jieying Chen ${ }^{1}$, Michel Ludwig ${ }^{2}$, Yue $\mathrm{Ma}^{3}$, and Dirk Walther ${ }^{4}$ \\ ${ }^{1}$ Laboratoire de Recherche en Informatique, Université Paris-Saclay, France \\ jieying.chen@lri.fr, https : //orcid.org/0000-0002-2497-645X \\ ${ }^{2}$ Luxembourg, michel.ludwig@gmail.com, \\ https://orcid.org/0000-0002-1899-5777 \\ ${ }^{3}$ Laboratoire de Recherche en Informatique, Université Paris-Saclay, France \\ yue.ma@lri.fr, https ://orcid.org/0000-0002-2805-2473 \\ ${ }^{4}$ Fraunhofer IVI, Dresden, Germany \\ dirk.walther@ivi.fraunhofer.de, https://orcid.org/0000-0002-5509-8899
}

\begin{abstract}
For the development of large-scale representations of knowledge, the application of methodologies and design principles becomes relevant. The knowledge may be organized in ontologies in a modular and hierarchical fashion. An upper-level (reference) ontology typically provides specifications of requirements, functions, design or standards that are to be complied with by domain ontologies for a specific task on a lower level (task ontology) in the hierarchy. Verifying whether and how specifications have been implemented by a task ontology becomes a challenge when relevant axioms of the domain ontology need to be inspected. We consider specifications to be defined using entailments of certain queries over a given vocabulary. For selecting the relevant axioms from task ontologies, we propose a novel module notion called projection module that entails the queries that follow from a reference ontology. We develop algorithms for computing minimal projection modules of Description Logic terminologies for subsumption, instance and conjunctive queries.
\end{abstract}

\section{Introduction}

A common practice in the area of the Semantic Web is to reuse and extend existing ontologies for a specific task. Therefore, an approach to comparing multiple ontologies is often desired. In this paper, we propose the notion of projection module which characterizes the relative knowledge of an ontology, say, an ontology developed for a specific task (called task ontology), by taking another ontology as a reference (called reference ontology), e.g. an upper-level ontology. This can thus lead to (1) a method for comparing the entailment capacities of any two ontologies about a given vocabulary of interest, and (2) a fine-grained ontology comparison measurement between two ontologies.

\footnotetext{
* This work is partially funded by the ANR project GoAsQ (ANR-15-CE23-0022).
} 


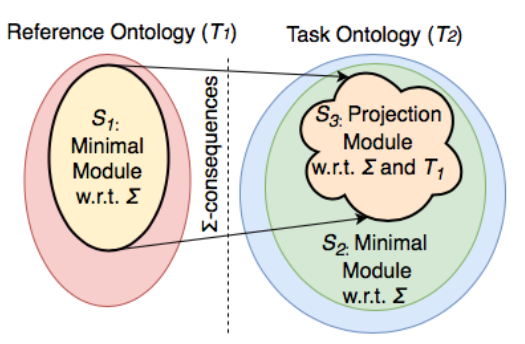

Fig. 1. Projection Module

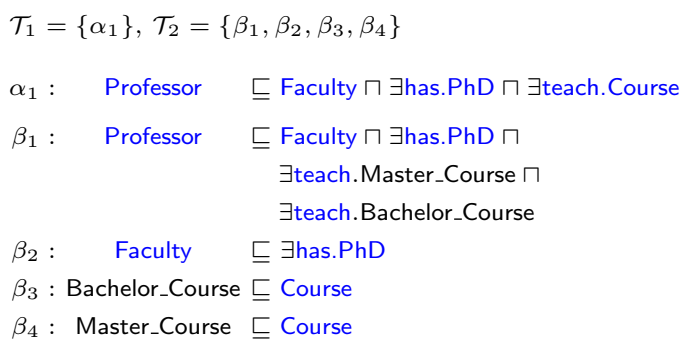

Fig. 2. Example: Minimal Projection Modules

As illustrated in Figure 1, for a user interest expressed as a set $\Sigma$ of concept and role names, the reference ontology $\mathcal{T}_{1}$ (resp. task ontology $\mathcal{T}_{2}$ ) contains $S_{1}$ (resp. $S_{2}$ ) as a sub-ontology, e.g., a minimal module $[9,10]$, that provides a minimal number of axioms entailing all $\Sigma$-consequences. A projection module, on the other hand, additionally takes a reference ontology into account as to preserve the relevant $\Sigma$-knowledge only, which can yield an even smaller module $S_{3}$ of $\mathcal{T}_{2}$. Figure 2 shows a concrete example with $\mathcal{T}_{1}$ being the upperlevel (reference) ontology modelling aspects of a university domain, and a task ontology $\mathcal{T}_{2}$ as an extended and modified version of $\mathcal{T}_{1}$. Consider the signature $\Sigma=\{$ Professor, Faculty, has, $\mathrm{PhD}$, teach, Course (consisting of the symbols marked in blue in Figure 2). Then $\mathcal{T}_{1}$ has merely one module $S_{1}=\left\{\alpha_{1}\right\}$ that preserves $\mathcal{T}_{1}$ 's knowledge about $\Sigma$. When extracting modules of $\mathcal{T}_{2}$ for $\Sigma$, we obtain $\mathcal{T}_{2}$ itself using existent module notions such as modules based on locality [14] or the module extracted by MEX [20]. Here we have two candidates for a minimal module $S_{2}$ of $\mathcal{T}_{2}$ that each preserve all inclusions over $\Sigma:\left\{\beta_{1}, \beta_{2}, \beta_{3}\right\}$ and $\left\{\beta_{1}, \beta_{2}, \beta_{4}\right\}[9]$. The projection modules of $\mathcal{T}_{2}$, however, preserving the $\Sigma$ inclusions entailed by $\mathcal{T}_{1}$ are even smaller with $S_{3}=\left\{\beta_{1}, \beta_{3}\right\}$ or $S_{3}=\left\{\beta_{1}, \beta_{4}\right\}$. Every projection module $S_{3}$ is a strict subset of a minimal module of $\mathcal{T}_{2}$, which is in line with the fact that the task ontology $\mathcal{T}_{2}$ has extended the reference ontology $\mathcal{T}_{1}$ with new $\Sigma$-consequences, e.g., Faculty $\sqsubseteq \exists$ has.PhD.

Various approaches to comparing ontologies have been suggested, including ontology matching [13] and logical difference [22, 23, 25, 27]. Ontology matching is the process of determining correspondences, e.g., the subsumption relations between two concept or role names from different ontologies, for which a good concept similarity $[1,26]$ is often helpful. In contrast, logical difference focuses on the comparison of entailed logical consequences from each ontology and returns difference witnesses if differences are present. When an ontology has no logical difference compared to another one, our approach further extracts sub-ontologies of the first that contain the knowledge as represented by the second ontology.

Ontology modularity $[9,18,22,25,28,29]$ is about the extraction of subontologies that preserve all logical consequences over a signature. The proposed projection module is different from modules of a single ontology, as illustrated by the example in Figure 2. To compute projection modules, in this paper, we generalize the notion of justification to the notion of subsumption justification as a minimal set of axioms that maintains the entailment of a conse- 
quence. Our algorithm employs the classical notion of justification to compute subsumption justifications. Currently, the approaches for computing all justifications of an ontology for a consequence can be classified into two categories: "glass-box" $[2,5,16,17]$ and "black-box" $[11,16,30]$.

We proceed as follows. After reviewing some preliminaries in Section 2, the notion of a minimal project module for subsumption, instance and conjunctive queries is introduced in Section 3. In Section 4, we introduce the algorithm for computing minimal projection modules. In Section 5, two applications of minimal projection modules are presented. Finally, we close the paper with a conclusion in Section 6.

\section{Preliminaries}

We start by reviewing the description logic $\mathcal{E} \mathcal{L}$ and several of its extensions.

Let $N_{C}, N_{R}$ and $N_{I}$ be mutually disjoint and countably infinite sets of concept names, role names and instance names. The signature $\operatorname{sig}(\xi)$ is the set of concept and role names occurring in $\xi$, where $\xi$ ranges over any syntactic object. The sets of $\mathcal{E} \mathcal{L}$-concepts $C, \mathcal{E} \mathcal{L}^{\text {ran }}$-concepts $D, \mathcal{E} \mathcal{L}^{\sqcap}$-concepts $E$, and $\mathcal{E} \mathcal{L}^{\sqcap, u}$-concepts $F$, and the sets of $\mathcal{E} \mathcal{L} \mathcal{H}^{r}$-inclusions $\alpha, \mathcal{E} \mathcal{L}^{\text {ran }}$-inclusions $\beta$ and $\mathcal{E} \mathcal{L}^{\text {ran, } \sqcap, u}$-inclusions $\gamma$ are built according to the grammar rules:

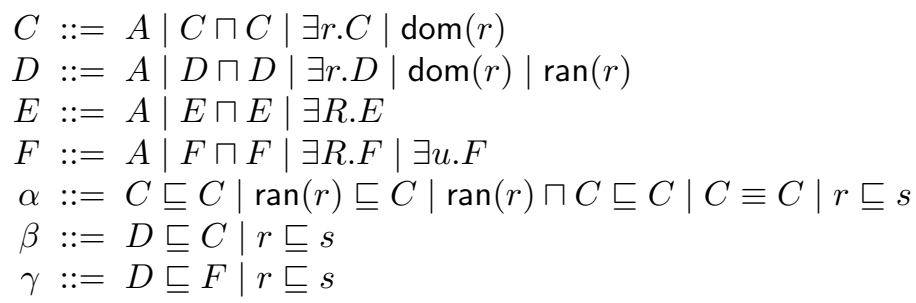

where $A \in \mathrm{N}_{\mathrm{C}}, r, s \in \mathrm{N}_{\mathrm{R}}, u$ is a fresh logical symbol (the universal role) and $R=r_{1} \sqcap \ldots \sqcap r_{n}$ with $r_{1}, \ldots, r_{n} \in \mathrm{N}_{\mathrm{R}}$, for $n \geq 1$. We refer to inclusions also as axioms. A $\Gamma$-TBox is a finite set of $\Gamma$-inclusions, where $\Gamma$ ranges over the sets of $\mathcal{E} \mathcal{L} \mathcal{H}^{r}$ - and $\mathcal{E} \mathcal{L}^{\text {ran, } \sqcap, u}$-inclusions. We use $\operatorname{lhs}(\alpha)$ (resp. $\left.\operatorname{rhs}(\alpha)\right)$ to represent the left-hand side (resp. right-hand side) of an inclusion $\alpha$.

The semantics is defined as usual in terms of interpretations interpreting concept/role names as unary/binary relations and are then inductively extended to complex concepts. The notions of satisfaction of a concept, axiom and TBox as well as the notions of a model and the logical consequence relation are defined as usual [4].

An $\mathcal{E} \mathcal{L} \mathcal{H}^{r}$-terminology $\mathcal{T}$ is an $\mathcal{E} \mathcal{L} \mathcal{H}^{r}$-TBox consisting of axioms $\alpha$ of the form $A \sqsubseteq C, A \equiv C, r \sqsubseteq s, \operatorname{ran}(r) \sqsubseteq C$ or $\operatorname{dom}(r) \sqsubseteq C$, where $A$ is a concept name, $C$ an $\mathcal{E} \mathcal{L}$-concept and no concept name occurs more than once on the left-hand side of an axiom. To simplify the presentation we assume that terminologies do not contain axioms of the form $A \equiv B$ or $A \equiv \top$ (after having removed multiple T-conjuncts) for concept names $A$ and $B$. For a terminology $\mathcal{T}$, let $\prec \mathcal{T}$ be a binary relation over $\mathrm{N}_{\mathrm{C}}$ satisfying that $A \prec \mathcal{T} B$ iff there is an axiom of the form 
$A \sqsubseteq C$ or $A \equiv C$ in $\mathcal{T}$ such that $B \in \operatorname{sig}(C)$. A terminology $\mathcal{T}$ is acyclic if the transitive closure $\prec_{\mathcal{T}}^{+}$of $\prec_{\mathcal{T}}$ is irreflexive; otherwise $\mathcal{T}$ is cyclic. We say that a concept name $A$ is conjunctive in $\mathcal{T}$ iff there exist concept names $B_{1}, \ldots, B_{n}$, $n>0$, such that $A \equiv B_{1} \sqcap \ldots \sqcap B_{n} \in \mathcal{T}$; otherwise $A$ is said to be non-conjunctive in $\mathcal{T}$. An $\mathcal{E} \mathcal{L} \mathcal{H}^{r}$-terminology $\mathcal{T}$ is normalised iff it only contains axioms of the forms

$$
\begin{aligned}
& -r \sqsubseteq s, \varphi \sqsubseteq B_{1} \sqcap \ldots \sqcap B_{n}, A \sqsubseteq \exists r . B, A \sqsubseteq \operatorname{dom}(r), \text { and } \\
& -A \equiv B_{1} \sqcap \ldots \sqcap B_{m}, A \equiv \exists r . B,
\end{aligned}
$$

where $\varphi \in\{A, \operatorname{dom}(s), \operatorname{ran}(s)\}, n \geq 1, m \geq 2, A, B, B_{i} \in \mathrm{N}_{\mathrm{C}}, r, s \in \mathrm{N}_{\mathrm{R}}$, and each conjunct $B_{i}$ is non-conjunctive in $\mathcal{T}$. Every $\mathcal{E} \mathcal{L} \mathcal{H}^{r}$-terminology $\mathcal{T}$ can be normalised in polynomial time such that the resulting terminology is a conservative extension of $\mathcal{T}$ [19]. A subset $M \subseteq \mathcal{T}$ is called a justification for an $\mathcal{E} \mathcal{L H}$-concept inclusion $\alpha$ from $\mathcal{T}$ iff $M \models \alpha$ and $M^{\prime} \not \models \alpha$ for every $M^{\prime} \subsetneq M$.

We denote the set of all justifications for an $\mathcal{E} \mathcal{L H}$-concept inclusion $\alpha$ from an $\mathcal{E} \mathcal{L} \mathcal{H}$-terminology $\mathcal{T}$ with Just $\mathcal{T}(\alpha)$. The latter may contain exponentially many justifications in the number of axioms in $\mathcal{T}$. An ABox contains assertions of the form $\mathrm{T}(a), A(a)$ and $r(a, b)$, where $a, b \in \mathrm{N}_{\mathrm{I}}$ and $r \in \mathrm{N}_{\mathrm{R}}$. An $A B o x$ consists of finitely many ABox assertions.

Let $N_{I}$ and $N_{V}$ be disjoint sets of individual and variable names. A conjunctive query is a first-order formula built according to the following format: $\exists y_{1} \ldots \exists y_{n} \cdot \bigwedge_{i \in I_{1}} A_{i}\left(s_{i}\right) \wedge \bigwedge_{j \in I_{2}} r_{j}\left(t_{j}, t_{j}^{\prime}\right)$, where $y_{1}, \ldots, y_{n} \in N_{\vee}$ for $n \geq 1$ are variable names, $I_{1}, I_{2}$ are finite sets of indices, and for $i \in I_{1}$ and $j \in I_{2}, A_{i}$ ranges over concept names in $\mathrm{N}_{\mathrm{C}}, r_{j}$ ranges over role names in $\mathrm{N}_{\mathrm{R}}$, and $s_{i}, t_{j}, t_{j}^{\prime}$ range over individual and variable names in $N_{1} \cup N_{V}$.

A signature $\Sigma$ is a finite set of symbols from $\mathrm{N}_{\mathrm{C}}$ and $\mathrm{N}_{\mathrm{R}}$. The symbol $\Sigma$ is used as a subscript to sets of concepts or inclusions to denote that the elements only use symbols from $\Sigma$, e.g., $\mathcal{E} \mathcal{L} \mathcal{H}_{\Sigma}^{r}$ and $\mathcal{E} \mathcal{L}_{\Sigma}^{\text {ran, } \sqcap, u}$. For a signature $\Sigma$, let $\Sigma^{\text {dom }}=\left\{\operatorname{dom}(r) \mid r \in \mathrm{N}_{\mathrm{R}} \cap \Sigma\right\}$ and $\Sigma^{\mathrm{ran}}=\left\{\operatorname{ran}(r) \mid r \in \mathrm{N}_{\mathrm{R}} \cap \Sigma\right\}$ be the sets consisting of concepts of the form $\operatorname{dom}(r)$ and $\operatorname{ran}(r)$ for every role name $r$ in $\Sigma$, respectively. We recall the notion of logical difference for concept subsumption queries, instance queries and conjunctive queries from [19,24]. For a more detailed introduction to description logics, we refer to [3,4]. For latest results on logical inseparability see $[7,8,15]$, and for a survey on query inseparability, see [6].

Definition 1 (Logical Difference). The $\mathcal{L}$-subsumption query difference, for some logic $\mathcal{L}$, the instance and conjunctive query difference between $\mathcal{T}_{1}$ and $\mathcal{T}_{2}$ w.r.t. $\Sigma$ are the sets $\operatorname{cDiff}_{\Sigma}^{\mathcal{L}}\left(\mathcal{T}_{1}, \mathcal{T}_{2}\right)$, $\operatorname{iDiff}_{\Sigma}\left(\mathcal{T}_{1}, \mathcal{T}_{2}\right)$, and $\mathrm{qDiff}{ }_{\Sigma}\left(\mathcal{T}_{1}, \mathcal{T}_{2}\right)$, respectively, where

$-\varphi \in \operatorname{cDiff}_{\Sigma}^{\mathcal{L}}\left(\mathcal{T}_{1}, \mathcal{T}_{2}\right)$ iff $\varphi$ is an $\mathcal{L}$-inclusion, $\mathcal{T}_{1} \models \varphi$ and $\mathcal{T}_{2} \not \models \varphi ;$

- $(\mathcal{A}, \lambda) \in \operatorname{iDiff}_{\Sigma}\left(\mathcal{T}_{1}, \mathcal{T}_{2}\right)$ iff $\mathcal{A}$ is a $\Sigma$-ABox and $\lambda$ a $\Sigma$-instance assertion such that $\left(\mathcal{T}_{1}, \mathcal{A}\right) \models \lambda$ and $\left(\mathcal{T}_{2}, \mathcal{A}\right) \not \models \lambda$;

- $(\mathcal{A}, q(\boldsymbol{a})) \in \mathrm{qDiff}_{\Sigma}\left(\mathcal{T}_{1}, \mathcal{T}_{2}\right)$ iff $\mathcal{A}$ is a $\Sigma$-ABox and $q(\boldsymbol{a})$ a $\Sigma$-conjunctive query such that $\left(\mathcal{T}_{1}, \mathcal{A}\right) \models q(\boldsymbol{a})$ and $\left(\mathcal{T}_{2}, \mathcal{A}\right) \not \models q(\boldsymbol{a})$. 
According to [19], $\mathcal{L}$-subsumption queries for $\mathcal{L}=\mathcal{E} \mathcal{L}^{\text {ran }}$ and $\mathcal{L}=\mathcal{E} \mathcal{L}^{\text {ran, }, u}$ are sufficient to detect the absence of any instance query and conjunctive query differences, respectively. Therefore, we only consider how to detect $\mathcal{L}$-subsumption queries for $\mathcal{L}=\left\{\mathcal{E} \mathcal{L} \mathcal{H}^{r}, \mathcal{E} \mathcal{L}^{\text {ran }}, \mathcal{E} \mathcal{L}^{\text {ran, },, u}\right\}$. Let $\alpha_{\Sigma}^{\mathcal{L}}$ be an $\mathcal{L}$-inclusion that only uses symbols in $\Sigma$. We organise the $\Sigma$-symbols (and the domain and range concepts over role names from $\Sigma$ ) that occur as "witnesses" of a $\mathcal{L}$-subsumption query difference between $\mathcal{T}_{1}$ and $\mathcal{T}_{2}$ as follows:

$$
\mathrm{Wtn}_{\Sigma}^{\mathcal{L}}\left(\mathcal{T}_{1}, \mathcal{T}_{2}\right):=\left(\operatorname{roleWtn}{ }_{\Sigma}^{\mathcal{L}}\left(\mathcal{T}_{1}, \mathcal{T}_{2}\right), \operatorname{lhsW\operatorname {tn}} \mathcal{L}_{\Sigma}^{\mathcal{L}}\left(\mathcal{T}_{1}, \mathcal{T}_{2}\right), \operatorname{rhsWtn} \mathcal{L}_{\Sigma}^{\mathcal{L}}\left(\mathcal{T}_{1}, \mathcal{T}_{2}\right)\right)
$$

where $\operatorname{roleWtn} \mathcal{L}_{\Sigma}\left(\mathcal{T}_{1}, \mathcal{T}_{2}\right)=\left\{r \in \Sigma \cap \mathrm{N}_{\mathrm{R}} \mid r \sqsubseteq s\right.$ or $\left.s \sqsubseteq r \in \operatorname{cDiff}_{\Sigma}^{\mathcal{L}}\left(\mathcal{T}_{1}, \mathcal{T}_{2}\right)\right\}$, $\operatorname{lhsW} \operatorname{tn}_{\Sigma}^{\mathcal{L}}\left(\mathcal{T}_{1}, \mathcal{T}_{2}\right)=\left\{\varphi \in\left(\Sigma \cap \mathrm{N}_{\mathrm{C}}\right) \cup \Sigma^{\text {dom }} \cup \Sigma^{\mathrm{ran}} \mid \varphi \sqsubseteq \operatorname{rhs}\left(\alpha_{\Sigma}^{\mathcal{L}}\right)\right.$ and $\alpha$ is a $\mathcal{L}_{\Sigma}-$ inclusion $\}$ and $\operatorname{rhsWtn}{ }_{\Sigma}^{\mathcal{L}}\left(\mathcal{T}_{1}, \mathcal{T}_{2}\right)=\left\{A \in \Sigma \cap \mathrm{N}_{\mathrm{C}} \mid \operatorname{lhs}\left(\alpha_{\Sigma}^{\mathcal{L}}\right) \sqsubseteq A \in\right.$ $\left.\operatorname{cDiff}_{\Sigma}^{Q}\left(\mathcal{T}_{1}, \mathcal{T}_{2}\right)\right\}$. The set $\operatorname{Wtn}_{\Sigma}^{\mathcal{L}}\left(\mathcal{T}_{1}, \mathcal{T}_{2}\right)$ can be seen as a finite representation of the set $\operatorname{cDiff}{ }_{\Sigma}^{\mathcal{L}}\left(\mathcal{T}_{1}, \mathcal{T}_{2}\right)$, which is typically infinite when it is not empty. It follows from the "primitive witnesses" theorems in [19] that $\operatorname{cDiff}_{\Sigma}^{\mathcal{L}}\left(\mathcal{T}_{1}, \mathcal{T}_{2}\right)=\emptyset$ iff $\operatorname{Wtn}_{\Sigma}^{\mathcal{L}}\left(\mathcal{T}_{1}, \mathcal{T}_{2}\right)=(\emptyset, \emptyset, \emptyset)$. Thus, deciding the existence of logical differences is equivalent to decide non-emptiness of the three witness sets.

\section{$3 \quad$ Projection Modules}

To understand the relations among different ontologies, we introduce the notion of projection module, as a way to explain how the knowledge that is encoded in a reference ontology is implemented in a task ontology. We are interested in computing all projection modules, since it provides a complete list of all implementations of an ontology regarding a reference, each of which may be necessary to be checked. To enable a manual validation by domain experts, we need to present only necessary information, so we focus on computing minimal projection modules.

A terminology $\mathcal{T}_{1}$ together with a signature $\Sigma$ and a type of query $Q$ determine a set $\Phi$ of queries from $Q$ formulated using only symbols from $\Sigma$ that follow from $\mathcal{T}_{1}$. A projection module of another terminology $\mathcal{T}_{2}$ is a subset of $\mathcal{T}_{2}$ that entails the queries in $\Phi$. For convenience, we bundle the parameters together in a tuple $\rho=\left\langle\mathcal{T}_{1}, \Sigma, \mathcal{T}_{2}\right\rangle$, which we call a projection setting.

Definition 2 (Projection Module). Let $\rho=\left\langle\mathcal{T}_{1}, \Sigma, \mathcal{T}_{2}\right\rangle$ be a projection setting, $\mathcal{A}$ be a $\Sigma$-ABox. A subset $\mathcal{M} \subseteq \mathcal{T}_{2}$ is a subsumption (resp. instance, conjunctive) query projection module under projection setting $\rho$, denoted as $\mathcal{M}_{\rho}^{c}$ (resp. $\left.\mathcal{M}_{\rho}^{i}, \mathcal{M}_{\rho}^{q}\right)$ iff:

- $\mathcal{M}_{\rho}^{c}$ : for each $\mathcal{E} \mathcal{L} \mathcal{H}_{\Sigma}^{r}$-inclusion $\alpha$, if $\mathcal{T}_{1} \models \alpha$, then $\mathcal{M} \models \alpha$;

- $\mathcal{M}_{\rho}^{i}$ : for each $\Sigma$-instance assertion $\lambda$, if $\left(\mathcal{T}_{1}, \mathcal{A}\right) \models \lambda$, then $(\mathcal{M}, \mathcal{A}) \models \lambda$;

- $\mathcal{M}_{\rho}^{q}$ : for each $q(\boldsymbol{a})$, if $\left(\mathcal{T}_{1}, \mathcal{A}\right)=q(\boldsymbol{a})$, then $(\mathcal{M}, \mathcal{A}) \models q(\boldsymbol{a})$, where $\boldsymbol{a}$ is a tuple of individual names from $\mathcal{A}$ and $q(\boldsymbol{a})$ is a $\Sigma$-conjunctive query.

A minimal subsumption (resp. instance, conjunctive) query projection module is a projection module $\mathcal{M}_{\rho}^{c}$ (resp. $\mathcal{M}_{\rho}^{i}, \mathcal{M}_{\rho}^{q}$ ) minimal w.r.t. $\subsetneq$. 
Note that there may exist several, even exponentially many minimal projection modules. It can readily be checked that $\mathrm{cDiff}_{\Sigma}^{\mathcal{L}}\left(\mathcal{T}_{1}, \mathcal{M}_{\rho}^{c}\right)=\emptyset$, for $\mathcal{L}=\mathcal{E} \mathcal{L} \mathcal{H}^{r}$, $\operatorname{iDiff}_{\Sigma}\left(\mathcal{T}_{1}, \mathcal{M}_{\rho}^{i}\right)=\emptyset$ and $\mathrm{qDiff}_{\Sigma}\left(\mathcal{T}_{1}, \mathcal{M}_{\rho}^{q}\right)=\emptyset$ (cf. Definition 1).

Example 1. Suppose $\mathcal{T}_{1}=\left\{A_{1} \sqsubseteq A_{2}, A_{2} \sqsubseteq A_{3}\right\}, \mathcal{T}_{2}=\left\{A_{1} \sqsubseteq A_{3} \sqcap B_{1}, B_{1} \sqsubseteq\right.$ $\left.\exists r . A_{3}\right\}$, and the interested vocabulary $\Sigma=\left\{A_{1}, A_{3}, r\right\} . \mathcal{T}_{2}$ has no logical difference from $\mathcal{T}_{1}$. However, the concept project module of $\mathcal{T}_{2}$ with respect to $\mathcal{T}_{1}$ and $\Sigma$ is $\left\{A_{1} \sqsubseteq A_{3} \sqcap B_{1}\right\} \subsetneq \mathcal{T}_{2}$. This means that a strict sub-ontology of $\mathcal{T}_{2}$ is sufficient to capture all the information of $\mathcal{T}_{1}$ about $\Sigma$. Moreover, $\mathcal{T}_{2}$ also entails a consequence $A_{1} \sqsubseteq \exists r . A_{3}$, which is not the case for $\mathcal{T}_{1}$.

The following example shows that the three notions of projection modules based on different query languages are distinct.

Example 2. Let $\mathcal{T}=\left\{X \sqsubseteq Y, Y \sqsubseteq \exists t . Z, \operatorname{ran}(r) \sqsubseteq A_{1}, \operatorname{ran}(s) \sqsubseteq A_{2}, B \equiv A_{1} \sqcap A_{2}\right\}$, $\Sigma=\{X, Y, Z, B, r, s\}$, and $\rho=\langle\mathcal{T}, \sigma, \mathcal{T}\rangle$. We have that $\mathcal{M}_{\rho}^{c}=\{X \sqsubseteq Y\}$, $\mathcal{M}_{\rho}^{i}=\mathcal{M}_{\rho}^{c} \cup\left\{\operatorname{ran}(r) \sqsubseteq A_{1}, \operatorname{ran}(s) \sqsubseteq A_{2}, B \equiv A_{1} \sqcap A_{2}\right\}$ and $\mathcal{M}_{\rho}^{q}=\mathcal{T}$.

Definition 3. Let $Q \in\{c, i, q\}$. The relationship between $\mathcal{T}_{1}$ and $\mathcal{T}_{2}$ is a $\langle\Sigma, Q\rangle$ implementation, denoted $\mathcal{T}_{1} \rightsquigarrow{ }_{\Sigma}^{Q} \mathcal{T}_{2}$, iff there exists a projection module $\mathcal{M}_{\rho}^{Q}$ under the setting $\rho=\left\langle\mathcal{T}_{1}, \Sigma, \mathcal{T}_{2}\right\rangle$.

If $\mathcal{T}_{1} \rightsquigarrow{ }_{\Sigma}^{Q} \mathcal{T}_{2}$, we also say that $\mathcal{T}_{2}\langle\Sigma, Q\rangle$-implements $\mathcal{T}_{1}$. In case $\mathcal{T}_{1}$ and $\mathcal{T}_{2}\langle\Sigma, Q\rangle$ implement each other, they cannot be separated using the query language $Q$.

Proposition 1. Let $\mathcal{T}_{1} \rightsquigarrow{ }_{\Sigma}^{Q} \mathcal{T}_{2}$ and $\mathcal{T}_{2} \rightsquigarrow{ }_{\Sigma}^{Q} \mathcal{T}_{1}$. Then:

$-Q=c: \operatorname{cDiff}_{\Sigma}^{\mathcal{L}}\left(\mathcal{T}_{1}, \mathcal{T}_{2}\right)=\operatorname{cDiff}_{\Sigma}^{\mathcal{L}}\left(\mathcal{T}_{2}, \mathcal{T}_{1}\right)=\emptyset$, for $\mathcal{L}=\mathcal{E} \mathcal{L} \mathcal{H}^{r} ;$

- $Q=i: \operatorname{iDiff}_{\Sigma}\left(\mathcal{T}_{1}, \mathcal{T}_{2}\right)=\operatorname{iDiff}_{\Sigma}\left(\mathcal{T}_{2}, \mathcal{T}_{1}\right)=\emptyset$; and

$-Q=q: \operatorname{qDiff}_{\Sigma}\left(\mathcal{T}_{1}, \mathcal{T}_{2}\right)=\operatorname{qDiff}_{\Sigma}\left(\mathcal{T}_{2}, \mathcal{T}_{1}\right)=\emptyset$.

We obtain the following monotonicity properties of the $\langle\Sigma, Q\rangle$-implementation relation.

Proposition 2 (Implementation Monotonicity).

(i) If $\mathcal{T}_{2} \subseteq \mathcal{T}_{3}$ and $\mathcal{T}_{1} \rightsquigarrow \stackrel{Q}{\Sigma} \mathcal{T}_{2}$, then $\mathcal{T}_{1} \rightsquigarrow \stackrel{Q}{\Sigma} \mathcal{T}_{3}$;

(ii) If $\mathcal{T}_{1} \subseteq \mathcal{T}_{2}$ and $\mathcal{T}_{2} \rightsquigarrow{ }_{\Sigma} \mathcal{T}_{3}$, then $\mathcal{T}_{1} \rightsquigarrow{ }_{\Sigma} \mathcal{T}_{3}$.

Property $(i)$ states that if a terminology $\mathcal{T}_{3}$ is obtained from $\mathcal{T}_{2}$ by adding further axioms to it, then it $\langle\Sigma, Q\rangle$-implements all the terminologies $\mathcal{T}_{1}$ that $\mathcal{T}_{2}$ $\langle\Sigma, Q\rangle$-implements. Property $\left(\right.$ ii) states $\mathcal{T}_{3}\langle\Sigma, Q\rangle$-implements all subsets $\mathcal{T}_{1}$ of $\mathcal{T}_{2}$ provided that $\mathcal{T}_{3}\langle\Sigma, Q\rangle$-implements $\mathcal{T}_{2}$. We leave investigating certain robustness properties of $\rightsquigarrow \underset{\Sigma}{Q}$ regarding signature extensions and varying query languages for future work; see, e.g., [21].

\section{Computing Minimal Projection Modules}

It is shown in [19] that detecting concept inclusion differences formulated in $\mathcal{E} \mathcal{L}^{\text {ran }}$ and $\mathcal{E} \mathcal{L}^{\text {ran, } \sqcap, u}$ is equivalent to detecting a difference with instance and conjunctive queries, respectively. We therefore consider subsumption queries from $\mathcal{E} \mathcal{L}^{\text {ran }}$ and $\mathcal{E} \mathcal{L}^{\text {ran, } \sqcap, u}$ to compute minimal projection justifications for instance and conjunctive queries, respectively. 


\subsection{Definition of Subsumption Projection Justifications}

For computing minimal subsumption projection modules for subsumption queries, we introduce the notion of a subsumption projection justification between two terminologies. As the notion depends on several parameters, we organise them for better readability in a tuple $\chi$ of the form $\left\langle\mathcal{T}_{1}, X_{1}, \Sigma, \mathcal{T}_{2}, X_{2}, \mathcal{L}\right\rangle$, where $\mathcal{T}_{1}$ and $\mathcal{T}_{2}$ are normalised $\mathcal{E} \mathcal{L} \mathcal{H}^{r}$-terminologies, $\Sigma$ is a signature, $X_{1}, X_{2} \in \mathrm{N}_{\mathrm{C}} \cup$ $\left\{\operatorname{dom}(r), \operatorname{ran}(r) \mid r \in \mathrm{N}_{\mathrm{R}}\right\}$, and $\mathcal{L} \in\left\{\mathcal{E} \mathcal{L} \mathcal{H}^{r}, \mathcal{E} \mathcal{L}^{\text {ran }}, \mathcal{E} \mathcal{L}^{\text {ran, }, u}\right\}$.

To obtain subsumption modules, we use an operator ' $\otimes$ ' to combine sets of role, subsumee and subsumer projection justifications. Given a set $\mathcal{S}$ and sets of sets $\mathbb{S}_{1}, \mathbb{S}_{2} \subseteq 2^{\mathcal{S}}$, we define $\mathbb{S}_{1} \otimes \mathbb{S}_{2}:=\left\{\mathcal{S}_{1} \cup \mathcal{S}_{2} \mid \mathcal{S}_{1} \in \mathbb{S}_{1}, \mathcal{S}_{2} \in \mathbb{S}_{2}\right\}$. For instance, if $\mathbb{S}_{1}=\left\{\left\{\alpha_{1}, \alpha_{2}\right\},\left\{\alpha_{3}\right\}\right\}$ and $\mathbb{S}_{2}=\left\{\left\{\alpha_{1}, \alpha_{3}\right\},\left\{\alpha_{4}, \alpha_{5}\right\}\right\}$, then $\mathbb{S}_{1} \otimes \mathbb{S}_{2}=$ $\left\{\left\{\alpha_{1}, \alpha_{2}, \alpha_{3}\right\},\left\{\alpha_{1}, \alpha_{2}, \alpha_{4}, \alpha_{5}\right\},\left\{\alpha_{3}, \alpha_{4}, \alpha_{5}\right\},\left\{\alpha_{1}, \alpha_{3}\right\}\right\}$. For a set $\mathbb{M}$ of sets, we define a function Minimise $_{\subseteq}(\mathbb{M})$ as follows: $\mathcal{M} \in$ Minimise $_{\subseteq}(\mathbb{M})$ iff $\mathcal{M} \in \mathbb{M}$ and there does not exist a set $\mathcal{M}^{\prime} \in \mathbb{M}$ such that $\mathcal{M}^{\prime} \subsetneq \mathcal{M}$. Continuing with the example, Minimise $\subseteq\left(\mathbb{S}_{1} \otimes \mathbb{S}_{2}\right)=\left\{\left\{\alpha_{1}, \alpha_{3}\right\},\left\{\alpha_{1}, \alpha_{2}, \alpha_{4}, \alpha_{5}\right\},\left\{\alpha_{3}, \alpha_{4}, \alpha_{5}\right\}\right\}$.

Definition 4 (Subsumption Projection Justification). Let $\chi=\left\langle\mathcal{T}_{1}, X_{1}, \Sigma\right.$, $\left.\mathcal{T}_{2}, X_{2}, \mathcal{L}\right\rangle$. A set $\mathcal{M}$ is a subsumee module under $\chi$ iff $\mathcal{M} \subseteq \mathcal{T}_{2}$ and for every $\mathcal{L}_{\Sigma^{-}}$ inclusion $\alpha: \mathcal{T}_{1} \models \operatorname{lhs}(\alpha) \sqsubseteq X_{1}$ implies $\mathcal{M} \models \operatorname{lhs}(\alpha) \sqsubseteq X_{2}$; and $\mathcal{M}$ is a subsumer module under $\chi$ iff $\mathcal{M} \subseteq \mathcal{T}_{2}$ and for every $\mathcal{L}_{\Sigma^{-}}$inclusion $\alpha: \mathcal{T}_{1} \models X_{1} \sqsubseteq \operatorname{rhs}(\alpha)$ implies $\mathcal{M}=X_{2} \sqsubseteq \operatorname{rhs}(\alpha)$.

$\mathcal{M}$ is called a subsumption projection module under $\chi$ iff $\mathcal{M}$ is a subsumee and a subsumer projection module under $\chi$. A subsumee (subsumer, subsumption) projection justification under $\chi$ is a subsumee (resp. subsumer, subsumption) projection module under $\chi$ that is minimal w.r.t. $\subsetneq$.

We denote the set of all subsumee (resp. subsumer, subsumption) justifications under $\chi$ as $\mathbb{J}_{\chi}^{\leftarrow}\left(\right.$ resp. $\left.\mathbb{J}_{\chi}, \mathbb{J}_{\chi}\right)$, where $\chi=\left\langle\mathcal{T}_{1}, \varphi_{1}, \Sigma, \mathcal{T}_{2}, \varphi_{2}, \mathcal{L}\right\rangle$, and $\varphi_{1}, \varphi_{2} \in$ $\left(\mathrm{N}_{\mathrm{C}} \cap \Sigma\right) \cup \Sigma^{\mathrm{dom}} \cup \Sigma^{\mathrm{ran}}$.

Definition 5 (Role Subsumption Projection Justification). Let $\rho=\left\langle\mathcal{T}_{1}, \Sigma\right.$, $\left.\mathcal{T}_{2}\right\rangle$ be a projection setting. A set $\mathcal{M}$ is called a role subsumption module under $\rho$ iff $\mathcal{M} \subseteq \mathcal{T}_{2}$ and for every $r, s \in \mathrm{N}_{\mathrm{R}} \cap \Sigma, \mathcal{T}_{1} \models r \sqsubseteq s$ implies $\mathcal{M} \models r \sqsubseteq s$. A minimal role subsumption projection justification under $\rho$ is the role subsumption module under $\rho$ that is minimal w.r.t. $\subsetneq$.

We denote the set of all role subsumption projection justifications under $\rho$ as $\mathbb{J}_{\rho}^{R}$. The following lemma states how role subsumption projection justifications can be computed. The lemma can be shown using Definition 5 and the notion of justification.

Lemma 1. Let $\rho=\left\langle\mathcal{T}_{1}, \Sigma, \mathcal{T}_{2}\right\rangle$ be a projection setting.

$$
\mathbb{J}_{\rho}^{R}=\text { Minimize }_{\subseteq}\left(\bigotimes_{r, s \in \Sigma \cap N_{\mathrm{R}}, \mathcal{T}_{1} \models r \sqsubseteq s} \operatorname{Just}_{\mathcal{T}_{2}}(r \sqsubseteq s)\right)
$$

Using Definitions 1 and 4, we obtain the following lemma stating the absence of certain concept names, and domain and range concepts over role names as lefthand and right-hand difference witnesses between two terminologies $\mathcal{T}_{1}$ and $\mathcal{T}_{2}$. 
Lemma 2. Let $\varphi \in\left(\mathrm{N}_{\mathrm{C}} \cap \Sigma\right) \cup \Sigma^{\mathrm{dom}} \cup \Sigma^{\text {ran }}$ and let $A \in \Sigma \cap \mathrm{N}_{\mathrm{C}}$. Additionally, let $\chi=\left\langle\mathcal{T}_{1}, \varphi, \Sigma, \mathcal{T}_{2}, \varphi, \mathcal{L}\right\rangle, \chi^{\prime}=\left\langle\mathcal{T}_{1}, A, \Sigma, \mathcal{T}_{2}, A, \mathcal{L}\right\rangle$ and $\mathcal{L} \in\left\{\mathcal{E} \mathcal{L} \mathcal{H}^{r}, \mathcal{E} \mathcal{L}^{\text {ran }}, \mathcal{E L}^{\text {ran }, \sqcap, u}\right\}$. Then:

$-\varphi \notin \operatorname{lhsW} \operatorname{tn}_{\Sigma}^{\mathcal{L}}\left(\mathcal{T}_{1}, J_{\varphi}\right)$ for every $J_{\varphi} \in \mathbb{J}_{\chi} \rightarrow$

- $A \notin \operatorname{rhsWtn} \mathcal{L}_{\Sigma}^{\mathcal{L}}\left(\mathcal{T}_{1}, J_{A}\right)$ for every $J_{A} \in \mathbb{J}_{\chi^{\prime}}^{\leftarrow}$;

- roleWtn ${ }_{\Sigma}^{\mathcal{L}}\left(\mathcal{T}_{1}, J\right)=\emptyset$ for every $J \in \mathbb{J}_{\rho}^{R}$.

We need at least one subsumption justification, for every potential difference witness, to be contained in a projection module in order to prevent the witness; cf. Lemma 2. This is made precise in the following theorem.

Theorem 1. Let $\rho=\left\langle\mathcal{T}_{1}, \Sigma, \mathcal{T}_{2}\right\rangle$ be a projection setting and let $Q \in\{c, i, q\}$. Additionally, let $\mathbb{M}_{\rho}^{Q}$ be the set of all minimal projection modules under $\rho$ for query type $Q$. Finally, let

$$
\mathbb{S}_{\rho}^{\mathcal{L}}=\operatorname{Minimize}_{\subseteq}\left(\mathbb{J}_{\rho}^{R} \otimes \bigotimes_{\varphi \in\left(\Sigma \cap N_{\mathcal{C}}\right) \cup \Sigma^{\operatorname{dom}} \cup \Sigma^{\text {ran }}} \underset{\mathbb{J}_{\chi(\varphi, \mathcal{L})}^{\rightarrow}}{\rightarrow} \bigotimes_{A \in \Sigma \cap \mathrm{N}_{\mathcal{C}}} \mathbb{J}_{\chi(A, \mathcal{L})}^{\leftarrow}\right)
$$

where $\chi(\psi, \mathcal{L})=\left\langle\mathcal{T}_{1}, \psi, \Sigma, \mathcal{T}_{2}, \psi, \mathcal{L}\right\rangle$, and $\mathcal{L}=\mathcal{E} \mathcal{L} \mathcal{H}^{r}$ if $Q=c, \mathcal{L}=\mathcal{E} \mathcal{L}^{\text {ran }}$ if $Q=i$, and $\mathcal{L}=\mathcal{E} \mathcal{L}^{\text {ran, } \sqcap, u}$ if $Q=q$. Then it holds that $\mathbb{M}_{\rho}^{Q}=\mathbb{S}_{\rho}^{\mathcal{L}}$.

In this paper, we present an algorithm for computing subsumee projection justifications for subsumption queries of $\mathcal{E} \mathcal{L}^{\text {ran, } \sqcap, u}$. Recall that $\mathcal{E} \mathcal{L}^{\text {ran, },, u}$ inclusions are sufficient to detect any difference that is detectable with conjunctive queries. The algorithm for computing subsumer projection justifications, and the algorithms for the other query languages are similar.

\subsection{Computing Subsumee Projection Justifications}

We now present the algorithm for computing subsumee projection justifications. The basic idea of the algorithm is to collect as few axioms from a terminology $\mathcal{T}_{2}$ as possible while ensuring the existence of a so-called subsumee simulation between another terminology $\mathcal{T}_{1}$ and $\mathcal{T}_{2}[12,27]$.

Inclusions of the form $\operatorname{ran}(r) \sqsubseteq X$ might cause non-trivial entailments. For example, let $\mathcal{T}_{1}=\left\{X \equiv \exists r . Y, Y \equiv A_{1} \sqcap A_{2}\right\}$ and $\Sigma=\left\{X, A_{1}, A_{2}, r\right\}$. Then $\mathcal{T}_{1}$ entails that $X$ is subsumed by the $\Sigma$-concepts $\exists r . \top$ and $\exists r .\left(A_{1} \sqcap A_{2}\right)$ (modulo equivalence). For $\mathcal{T}_{2}=\mathcal{T}_{1} \cup\left\{\operatorname{ran}(r) \sqsubseteq A_{1}\right\}$, however, we additionally obtain $\mathcal{T}_{2}=$ $\exists r . A_{2} \sqsubseteq X$. Hence, when formulating the algorithms for computing subsumee simulations, an additional parameter $\zeta \in\{\epsilon\} \cup\left(\mathrm{N}_{\mathrm{R}} \cap \Sigma\right)$ is needed which is used in range concepts of the form $\operatorname{ran}(\zeta)$. We call this parameter context of a role. We treat $\epsilon$ as a special role name and set $\operatorname{ran}(\epsilon)=T$. The set of all role contexts, in symbols $\mathcal{C}^{\Sigma}$, is defined as $\mathcal{C}^{\Sigma}=\{\epsilon\} \cup\left(\mathrm{N}_{\mathrm{R}} \cap \Sigma\right)$.

To identify concept and role names that are relevant for a subsumee simulation that we propose later, we first use the following notion of $\Sigma$-entailment:

- $A \in \mathrm{N}_{\mathrm{C}}$ is $\Sigma$-entailed in $\mathcal{T}$ iff there is an $\mathcal{E} \mathcal{L}_{\Sigma}^{\text {ran }}$-concept $C$ such that $\mathcal{T}=$ $C \sqsubseteq A ;$ 

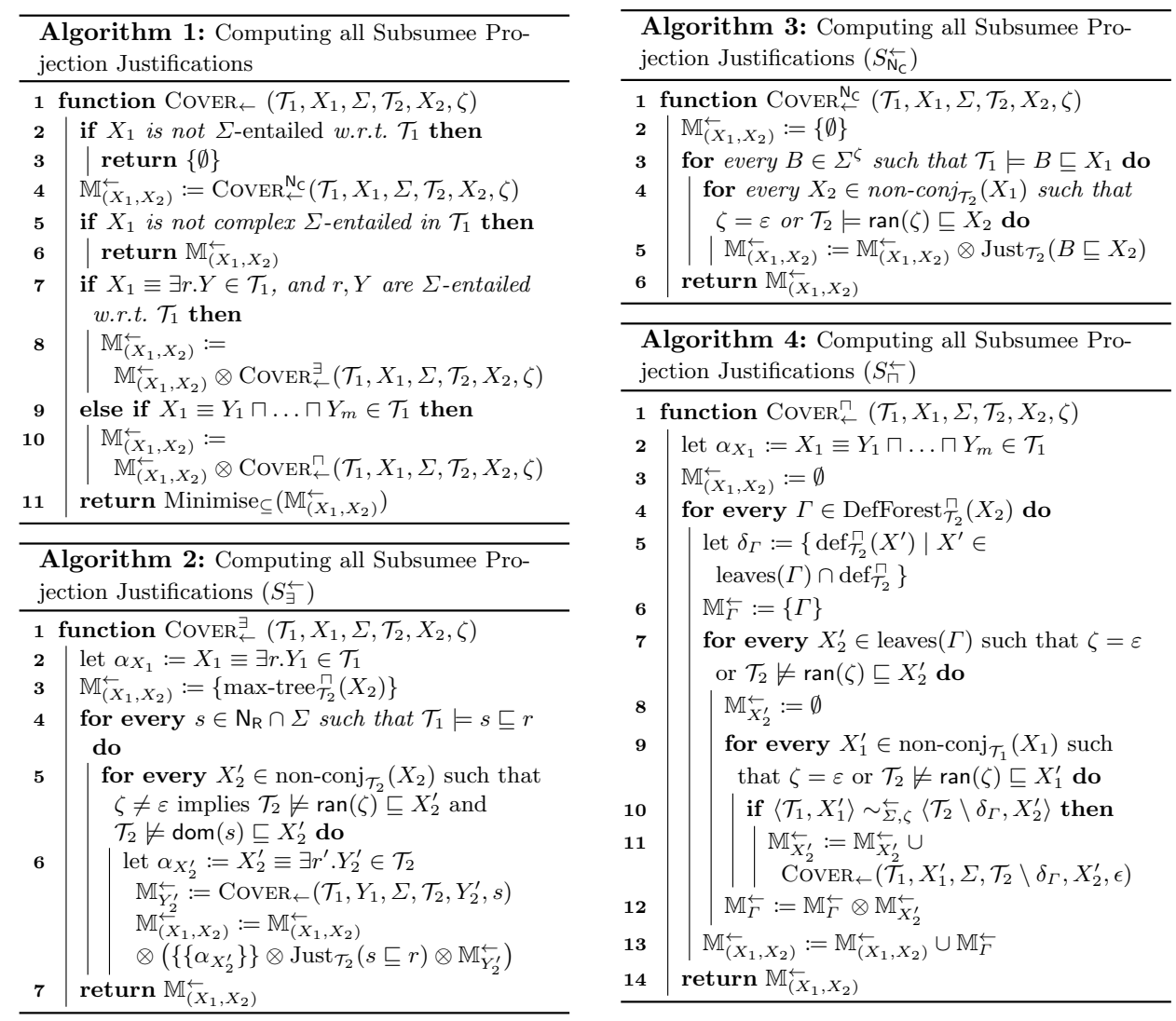

Fig. 3. Algorithms for Computing all Subsumee Justifications

- $s \in \mathrm{N}_{\mathrm{R}}$ is $\Sigma$-entailed in $\mathcal{T}$ iff there exists $s^{\prime} \in \mathrm{N}_{\mathrm{R}} \cap \Sigma$ such that $\mathcal{T} \models s^{\prime} \sqsubseteq s$;

- $A \in \mathrm{N}_{\mathrm{C}}$ is $(\Sigma, s)$-entailed in $\mathcal{T}$ iff there is an $\mathcal{E} \mathcal{L}_{\Sigma}^{\text {ran }}$-concept $C$ such that $\mathcal{T}=C \sqcap \operatorname{ran}(s) \sqsubseteq A$.

Moreover, we say that $X \in \mathrm{N}_{\mathrm{C}}$ is complex $\Sigma$-entailed w.r.t. $\mathcal{T}$ iff for every

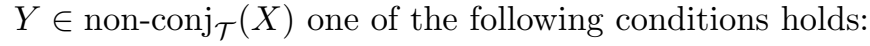

- there exists $B \in \Sigma$ such that $\mathcal{T} \models B \sqsubseteq Y$ and $\mathcal{T} \not \models B \sqsubseteq X$;

- there exists $Y \equiv \exists r . Z \in \mathcal{T}$ and $r, Z$ are $\Sigma$-entailed in $\overline{\mathcal{T}}$.

$X$ is said to be simply $\Sigma$-entailed if $X$ is $\Sigma$-entailed but not complex $\Sigma$-entailed. For example, let $\mathcal{T}=\left\{X \equiv X_{1} \sqcap X_{2}, B_{1} \sqsubseteq X_{1}, X_{2} \equiv \exists r . Z, B_{2} \sqsubseteq Z, s \sqsubseteq r\right\}$. We have that non-conj $\mathcal{T}_{\mathcal{T}}(X)=\left\{X_{1}, X_{2}\right\}$, then $r$ is $\Sigma$-entailed w.r.t. $\mathcal{T} ; X$ is complex $\Sigma$-entailed w.r.t. $\mathcal{T}$ for $\Sigma=\left\{B_{1}, B_{2}, s\right\}$; but $X$ is not complex $\Sigma^{\prime}$ entailed w.r.t. $\mathcal{T}$, where $\Sigma^{\prime}$ ranges over $\left\{B_{1}, B_{2}\right\},\left\{B_{1}, s\right\},\left\{B_{2}, s\right\}$. Additionally, $X$ is not complex $\Sigma$-entailed w.r.t. $\mathcal{T} \cup\left\{B_{1} \sqsubseteq X\right\}$. 
We now define the notion of a subsumee simulation from $\mathcal{T}_{1}$ to $\mathcal{T}_{2}$ as a subset of $\left(\mathrm{N}_{\mathrm{C}} \cap \operatorname{sig}\left(\mathcal{T}_{1}\right)\right) \times\left(\mathrm{N}_{\mathrm{C}} \cap \operatorname{sig}\left(\mathcal{T}_{2}\right)\right) \times \mathcal{C}_{\mathcal{T}_{1}}^{\Sigma}$, where $\mathcal{C}_{\mathcal{T}_{1}}^{\Sigma}:=\{\epsilon\} \cup\left(\mathrm{N}_{\mathrm{R}} \cap\left(\Sigma \cup \operatorname{sig}\left(\mathcal{T}_{1}\right)\right)\right)$ is the range of role contexts.

Definition 6 (Subsumee Simulation). A relation $S \subseteq \operatorname{sig}^{\mathrm{N}_{C}}\left(\mathcal{T}_{1}\right) \times \operatorname{sig}^{\mathrm{N}_{C}}\left(\mathcal{T}_{2}\right) \times$ $\mathcal{C}_{\mathcal{T}_{1}}^{\Sigma}$ is a $\Sigma$-subsumee simulation from $\mathcal{T}_{1}$ to $\mathcal{T}_{2}$ iff the following conditions hold:

$\left(S_{\mathrm{N}_{\mathrm{C}}}^{\leftarrow}\right)$ if $\left(X_{1}, X_{2}, \zeta\right) \in S$, then for every $\varphi \in \Sigma^{\zeta}$ and for every $X_{2}^{\prime} \in$ non-conj $j_{\mathcal{T}_{2}}\left(X_{2}\right)$ with $\mathcal{T}_{2} \mid \models \operatorname{ran}(\zeta) \sqsubseteq X_{2}^{\prime}, \mathcal{T}_{1} \models \varphi \sqsubseteq X_{1}$ implies $\mathcal{T}_{2} \models \varphi \sqsubseteq X_{2}^{\prime}$;

$\left(S_{\exists}^{\leftarrow}\right)$ if $\left(X_{1}, X_{2}, \zeta\right) \in S$ and $X_{1} \equiv \exists r . Y_{1} \in \mathcal{T}_{1}$ such that $\mathcal{T}_{1} \models s \sqsubseteq r$ for $s \in \Sigma$ and $Y_{1}$ is $(\Sigma, s)$-entailed in $\mathcal{T}_{1}$, then for every $X_{2}^{\prime} \in$ non-conj $j_{\mathcal{T}_{2}}\left(X_{2}\right)$ not entailed by $\operatorname{dom}(s)$ or $\operatorname{ran}(\zeta)$ w.r.t. $\mathcal{T}_{2}$, there exists $X_{2}^{\prime} \equiv \exists r^{\prime} . Y_{2} \in \mathcal{T}_{2}$ such that $\mathcal{T}_{2}=s \sqsubseteq r^{\prime}$ and $\left(Y_{1}, Y_{2}, s\right) \in S$;

$\left(S_{\Pi}^{\leftarrow}\right)$ if $\left(X_{1}, X_{2}, \zeta\right) \in S$ and $X_{1} \equiv Y_{1} \sqcap Y_{2} \sqcap \ldots \sqcap Y_{n} \in \mathcal{T}_{1}$, then for every $Y_{2} \in$ non-conj $j_{\mathcal{T}_{2}}\left(X_{2}\right)$ not entailed by $\operatorname{ran}(\zeta)$ w.r.t. $\mathcal{T}_{2}$, there exists $Y_{1} \in$ non-conj $\mathcal{T}_{1}\left(X_{1}\right)$ not entailed by $\operatorname{ran}(\zeta)$ w.r.t. $\mathcal{T}_{2}$ with $\left(Y_{1}, Y_{2}, \epsilon\right) \in S$.

We write $\mathcal{T}_{1} \sim \overleftarrow{\Sigma} \mathcal{T}_{2}$ iff there is a $\Sigma$-subsumee simulation $S$ from $\mathcal{T}_{1}$ to $\mathcal{T}_{2}$ such that for every $A \in \mathrm{N}_{\mathrm{C}} \cap \Sigma:(A, A, \epsilon) \in S$.

For $\zeta \in \Sigma \cap \mathrm{N}_{\mathrm{R}}$, we write $\left\langle\mathcal{T}_{1}, X_{1}\right\rangle \sim \overleftarrow{\Sigma}_{\zeta \zeta}\left\langle\mathcal{T}_{2}, X_{2}\right\rangle$ iff there is a $\Sigma$-subsumee simulation $S$ from $\mathcal{T}_{1}$ to $\mathcal{T}_{2}$ with $\left(X_{1}, X_{2}, \zeta\right) \in S$ for which $\mathcal{T}_{1} \sim \overleftarrow{\Sigma} \mathcal{T}_{2}$

A subsumee simulation captures the set of subsumees in the sense that $\mathcal{T}_{1} \sim \overleftarrow{\Sigma}$ $\mathcal{T}_{2}$ iff $\operatorname{rhsWtn}_{\Sigma}\left(\mathcal{T}_{1}, \mathcal{T}_{2}\right)=\emptyset$. Moreover, if a concept name $X_{2}$ in $\mathcal{T}_{2} \Sigma$-subsumee simulates a concept name $X_{1}$ in $\mathcal{T}_{1}$, then $X_{2}$ subsumes all $\Sigma$-concepts w.r.t. $\mathcal{T}_{2}$ that are subsumed by $X_{1}$ w.r.t. $\mathcal{T}_{1}$. Formally: $\left\langle\mathcal{T}_{1}, X_{1}\right\rangle \sim \overleftarrow{\Sigma, \zeta}_{\zeta}\left\langle\mathcal{T}_{2}, X_{2}\right\rangle$ iff for every $C \in \mathcal{E}_{\mathcal{L}_{\Sigma}^{\text {ran }}}: \mathcal{T}_{1}=C \sqsubseteq X_{1}$ implies $\mathcal{T}_{2}=C \sqsubseteq X_{2}[27]$.

Algorithm 1 provides the function Cover $\leftarrow$ for computing the set of all subsumee justifications. The algorithm recursively computes sets of axioms sufficient to construct a subsumee simulation. For better readability, the algorithm is structured into several parts, one for each condition of a subsumee simulation, cf. Definition 6. Algorithm 3 handles Case $\left(S_{N_{c}}^{\overleftarrow{C}}\right)$, Algorithm 2 takes care of Case $\left(S_{\exists}^{\leftarrow}\right)$ and Algorithm 4 is responsible for Case $\left(S_{\Pi}^{\leftarrow}\right)$. Note that each of these algorithms requires a role context $\zeta$ as an input parameter. The notion of complex $\Sigma$-entailment is employed in Algorithm 1. If $X$ is not complex $\Sigma$-entailment, then neither the existential nor the conjunctive case need to be considered, and Algorithm 1 terminates in Line 6.

Compared with computing subsumer projection justifications, the challenge of computing subsumee projection justifications is to handle conjunctions on the left-hand side of subsumptions. Concept names defined as conjunctions in $\mathcal{T}_{2}$ use conjuncts which in turn may also be defined as conjunctions. Such axioms form tree structures. When selecting axioms, all minimal subsets of $\mathcal{T}_{2}$, i.e., all sub-trees, that maintain a subsumee simulation need to be considered. To this end, we define for each concept name $X$ a so-called definitorial forest consisting of sets of axioms of the form $Y \equiv Y_{1} \sqcap \ldots \sqcap Y_{n}$ which can be thought of as forming trees. Any $\langle X, \Sigma\rangle$-subsumee projection justification contains the axioms of a selection of these trees, i.e., one tree for every conjunction formulated over 
$\Sigma$ that entails $X$ w.r.t. $\mathcal{T}$. Formally, we define a set of a $\operatorname{DefForest~}_{\mathcal{T}}^{\square}(X) \subseteq 2^{\mathcal{T}}$ to be the smallest set closed under the following conditions:

$-\emptyset \in \operatorname{DefForest}_{\mathcal{T}}^{\square}(X)$

- $\{\alpha\} \in \operatorname{DefForest}_{\mathcal{T}}^{\square}(X)$ for $\alpha:=X \equiv X_{1} \sqcap \ldots \sqcap X_{n} \in \mathcal{T}$; and

- $\Gamma \cup\{\alpha\} \in \operatorname{DefForest}_{\mathcal{T}}^{\sqcap}(X)$ for $\Gamma \in \operatorname{DefForest~}_{\mathcal{T}}^{\sqcap}(X)$ with $Z \equiv Z_{1} \sqcap \ldots \sqcap Z_{k} \in \Gamma$ and $\alpha:=Z_{i} \equiv Z_{i}^{1} \sqcap \ldots \sqcap Z_{i}^{n} \in \mathcal{T}$.

Given a tree $\Gamma \in \operatorname{DefForest}_{\mathcal{T}}^{\square}(X)$ rooted at $X$, we use leaves $(\Gamma)$ to denote the set $\operatorname{sig}(\Gamma) \backslash\{X \in \operatorname{sig}(C) \mid X \equiv C \in \Gamma\}$ of leaves if $\Gamma \neq \emptyset$; and $\{X\}$ otherwise. We denote with max-tree ${ }_{\mathcal{T}}^{\square}(X)$ the set in $\operatorname{DefForest}_{\mathcal{T}}^{\square}(X)$ that is maximal w.r.t. $\subseteq$. Finally, we set non- $\operatorname{conj}_{\mathcal{T}}(X):=\operatorname{leaves}\left(\max -\operatorname{tree}_{\mathcal{T}}^{\square}(X)\right)$ to be the set of leaves of the maximal tree. For example, for $\mathcal{T}=\left\{\alpha_{1}, \alpha_{2}, \alpha_{3}\right\}$ with $\alpha_{1}:=X \equiv Y \sqcap Z, \alpha_{2}:=Y \equiv Y_{1} \sqcap Y_{2}$, and $\alpha_{3}:=Z \equiv Z_{1} \sqcap Z_{2}$, we obtain DefForest ${ }_{\mathcal{T}}^{\sqcap}(X)=\left\{\emptyset,\left\{\alpha_{1}\right\},\left\{\alpha_{1}, \alpha_{2}\right\},\left\{\alpha_{1}, \alpha_{3}\right\},\left\{\alpha_{1}, \alpha_{2}, \alpha_{3}\right\}\right\}$. Moreover, we have that leaves $\left(\left\{\alpha_{1}, \alpha_{3}\right\}\right)=\left\{Y, Z_{1}, Z_{2}\right\}$, $\max _{\text {-tree }}{ }_{\mathcal{T}}(X)=\left\{\alpha_{1}, \alpha_{2}, \alpha_{3}\right\}$, and non- $\operatorname{conj}_{\mathcal{T}}(X)=\left\{Y_{1}, Y_{2}, Z_{1}, Z_{2}\right\}$.

The definitorial forest is used to enumerate and find all trees for which Case $\left(S_{\sqcap}^{\leftarrow}\right)$ holds, which is done in Algorithm 4. The set non-conj $\mathcal{T}_{\mathcal{T}}(X)$, however, is also used in Algorithm 2, which we discuss next. The existence of axiom $\alpha_{X_{1}}:=X_{1} \equiv \exists r . Y_{1} \in \mathcal{T}_{1}$ in Line 2 of Algorithm 2 is guaranteed by Line 7 of Algorithm 1. The axiom $\alpha_{X_{2}^{\prime}}:=X_{2}^{\prime} \equiv \exists r^{\prime} . Y_{2}^{\prime} \in \mathcal{T}_{2}$ in Line 6 of Algorithm 2 exists as we assume that $\mathcal{T}_{2}, X_{2}$ subsumee-simulates $\mathcal{T}_{1}, X_{1}$ w.r.t. $\Sigma$. Moreover, there is at most one axiom $\alpha_{X_{1}} \in \mathcal{T}_{1}$ and at most one $\alpha_{X_{2}^{\prime}} \in \mathcal{T}_{2}$ as $\mathcal{T}_{1}$ and $\mathcal{T}_{2}$ are terminologies. The concept name $X_{2}$ may be defined as a conjunction in $\mathcal{T}_{2}$ whose conjuncts in turn may also be defined as a conjunction in $\mathcal{T}_{2}$ and so forth. In Line 3 all axioms forming the maximal resulting definitorial conjunctive tree are collected.

For the next algorithm, we define $\operatorname{def}_{\mathcal{T}}^{\sqcap}:=\left\{X \in \operatorname{sig}^{\mathrm{N}_{c}}(\mathcal{T}) \mid X \equiv Y_{1} \sqcap \ldots \sqcap Y_{n} \in\right.$ $\mathcal{T}\}$ to be the set of concept names that are conjunctively defined in $\mathcal{T}$. For every $X \in \operatorname{def}_{\mathcal{T}}^{\sqcap}$, we set $\operatorname{def}_{\mathcal{T}}^{П}(X):=\alpha$, where $\alpha=X \equiv Y_{1} \sqcap \ldots \sqcap Y_{n} \in \mathcal{T}$.

The axiom $\alpha_{X_{1}}:=X_{1} \equiv Y_{1} \sqcap \ldots \sqcap Y_{m} \in \mathcal{T}_{1}$ in Line 2 of Algorithm 4 is guaranteed to exist by Line 9 of Algorithm 4. In case $X_{2}$ is defined as a conjunction in $\mathcal{T}_{2}$, the pair consisting of $\mathcal{T}_{2}$ containing only a partial conjunctive tree rooted at $X_{2}$ and $X_{2}$ needs to be considered to be sufficient to subsumee simulate $\mathcal{T}_{1}, X_{1}$. Therefore Algorithm 4 considers every partial conjunctive tree $\Gamma$ from DefForest ${ }_{\mathcal{T}_{2}}\left(X_{2}\right)$ in Line 4 and removes the axioms in $\delta_{\Gamma}$ connecting the leaves of $\Gamma$ with the remaining conjunctive tree from $\mathcal{T}_{2}$ in lines 10 and 11.

The following theorem states that Algorithm 1 indeed computes the set of subsumee projection justifications. The proof establishes that Algorithm 1 computes all possible subsets of $\mathcal{T}_{2}$ that are minimal w.r.t. $\subsetneq$ while preserving one of the considered $\Sigma$-subsumee simulations from $\mathcal{T}_{1}$ to $\mathcal{T}_{2}$.

Theorem 2. Let $\chi=\left\langle\mathcal{T}_{1}, \varphi_{1}, \Sigma, \mathcal{T}_{2}, \varphi_{2}, \mathcal{E} \mathcal{L}^{\text {ran, } \sqcap, u}\right\rangle$, and $\varphi_{1}, \varphi_{2} \in\left(\mathrm{N}_{\mathrm{C}} \cap \Sigma\right) \cup$ $\left\{\operatorname{dom}(r), \operatorname{ran}(r) \mid r \in \mathrm{N}_{\mathrm{R}} \cap \Sigma\right\}$. Additionally, let $\mathbb{M}:=\operatorname{CoveR}_{\leftarrow}\left(\mathcal{T}_{1}, \varphi_{1}, \Sigma, \mathcal{T}_{2}, \varphi_{2}, \epsilon\right)$ using Algorithm 1. Then $\mathbb{M}$ is the set of all subsumee justifications under $\chi$. 
Algorithm 1 runs in exponential time in the number of axioms contained in the input terminologies, in the worst case. On the one hand, the algorithm uses justifications (see Line 6 of Alg. 2 and Line 5 of Alg. 3) whose number grows exponentially for role inclusions as well as concept name inclusions. The different justifications are each incorporated using the operator $\otimes$ resulting in possibly different subsumption justifications. The majority of the running time will be spent on computing justifications. Another source of exponential blowup is contained in Line 4 of Algorithm 4. The number of elements in the set DefForest $\underset{\mathcal{T}}{\square}(X)$ grows exponentially in $|\mathcal{T}|$. According to our experience so far, however, it seems plausible to assume that definitorial forests in practical ontologies remain rather small and, thus, they do not cause a serious slowdown of the algorithm.

\section{Application of Minimal Projection Modules}

In this section, we discuss two applications of minimal projection modules.

\subsection{Computing Minimal Query Modules}

We first define the minimal query modules for different queries.

Definition 7 (Query Module). $A$ set $M \subseteq \mathcal{T}$ is a subsumption (resp. instance, conjunctive) query module of $\mathcal{T}$, denoted as $M_{\Sigma}^{c}$ (resp. $\left.M_{\Sigma}^{i}, M_{\Sigma}^{q}\right)$

- $M_{\Sigma}^{c}$ : for each $\mathcal{E} \mathcal{L} \mathcal{H}_{\Sigma}^{r}$-inclusion $\alpha$, if $\mathcal{T} \models \alpha$, then $M \models \alpha$;

- $M_{\Sigma}^{i}$ : for each $\Sigma$-instance assertion $\lambda$, if $(\mathcal{T}, \mathcal{A}) \models \lambda$, then $(M, \mathcal{A}) \models \lambda$;

- $M_{\Sigma}^{q}$ : for each $q(\boldsymbol{a})$, if $(\mathcal{T}, \mathcal{A}) \models q(\boldsymbol{a})$, then $(M, \mathcal{A}) \models q(\boldsymbol{a})$, where $\boldsymbol{a}$ is a tuple of individual names from $\mathcal{A}$ and $q(\boldsymbol{a})$ is a $\Sigma$-conjunctive query.

A subsumption (resp. instance, conjunctive) query module is called a minimal subsumption (resp. instance, conjunctive) query module iff it is minimal w.r.t. $\subsetneq$.

In general, the reference and the implementing/task ontologies do not coincide. Intuitively, the task ontology $\mathcal{T}_{2}$ might contain more knowledge about $\Sigma$ than the reference ontology $\mathcal{T}_{1}$. The following lemma illustrates the relationship between minimal projection modules and minimal query modules.

Lemma 3. Let $Q \in\{c, i, q\}$ and $\rho=\left\{\mathcal{T}_{1}, \Sigma, \mathcal{T}_{2}\right\}$. Then: for every minimal projection module $\mathcal{M}_{\rho}^{Q}$ for a query type $Q$ and under a projection setting $\rho$, there exists a minimal $Q$-query module $M_{\Sigma}^{Q}$ of $\mathcal{T}_{2}$ such that $\mathcal{M}_{\rho}^{Q} \subseteq M_{\Sigma}^{Q}$.

Example 3 (Fig. 2 contd.). The minimal projection module of $\mathcal{T}_{2}$ under $\rho=$ $\left\{\mathcal{T}_{1}, \Sigma, \mathcal{T}_{2}\right\}$ is $\left\{\left\{\beta_{1}, \beta_{3}\right\},\left\{\beta_{1}, \beta_{4}\right\}\right\}$, for any query type $Q \in\{c, i, q\}$. The minimal $Q$-query module of $\mathcal{T}_{2}$ w.r.t. $\Sigma$ is $\left\{\left\{\beta_{1}, \beta_{2}, \beta_{3}\right\},\left\{\beta_{1}, \beta_{2}, \beta_{4}\right\}\right\}$. The minimal $Q$ query module of $\mathcal{T}_{1}$ is $\left\{\alpha_{1}\right\}$.

One solution for importing $\Sigma$-knowledge of a reference ontology to a task ontology is to import a minimal $Q$-query module of the reference ontology. However, one can see that if we include $\alpha_{1}$ to $\mathcal{T}_{2}$, then $\alpha_{1}$ repeats the $\Sigma$-knowledge that is already represented by $\beta_{1}$. Besides, the resulting ontology would not be a terminology anymore. 
Consider a special projection setting of the form $\langle\mathcal{T}, \Sigma, \mathcal{T}\rangle$, where the reference ontology $\mathcal{T}$ is also the implementing ontology. We denote such reflexive projection settings with $\rho^{\circlearrowleft}$. A projection module $\mathcal{M}$ of $\mathcal{T}$ under $\rho^{\circlearrowleft}$ for subsumption (resp. instance, conjunctive) queries is a subset of $\mathcal{T}$ that preserves the answers to $\Sigma$-concept subsumption (resp. instance, conjunctive) queries as given by $\mathcal{T}$. It can readily be verified that a minimal projection module under a reflexive projection setting coincides with a minimal module for the type of queries considered.

\subsection{Ontology Comparison Measure}

In existent methods for measuring the entailment capacity of a terminology about a signature $\Sigma$ for a query language, one can use logical difference. However, the following example shows that using logical difference can be not sufficient in some case.

Example 4. Let $\alpha_{1}:=A \sqsubseteq B_{1} \sqcap B_{2} \sqcap B_{3} \sqcap B_{4}, \alpha_{2}:=B_{1} \sqsubseteq B_{2} \sqcap B_{3}, \alpha_{3}:=B_{2} \sqsubseteq B_{4}$, $\alpha_{4}:=B_{3} \sqsubseteq B_{4}$. Let $\mathcal{T}_{1}=\left\{\alpha_{1}\right\}, \mathcal{T}_{2}=\left\{\alpha_{1}, \alpha_{2}\right\}, \mathcal{T}_{3}=\left\{\alpha_{1}, \alpha_{2}, \alpha_{3}, \alpha_{4}\right\}$ and $\Sigma=\left\{A, B_{1}, B_{2}, B_{3}, B_{4}\right\}$. We have that $\operatorname{Wtn}_{\Sigma}^{\mathcal{L}}\left(\mathcal{T}_{1}, \mathcal{T}_{2}\right)=\operatorname{Wtn}_{\Sigma}^{\mathcal{L}}\left(\mathcal{T}_{1}, \mathcal{T}_{3}\right)=(\emptyset, \emptyset, \emptyset)$, for $\mathcal{L} \in\left\{\mathcal{E} \mathcal{L} \mathcal{H}^{r}, \mathcal{E} \mathcal{L}^{\text {ran }}, \mathcal{E} \mathcal{L}^{\text {ran }, \sqcap, u}\right\}$.

In Example 4, the notion of logical difference cannot be used to distinguish between $\mathcal{T}_{2}$ and $\mathcal{T}_{3}$ w.r.t. $\Sigma$ as $\mathcal{T}_{2}$ and $\mathcal{T}_{3}$ preserve the $\Sigma$-knowledge w.r.t. $\mathcal{T}_{1}$. However, intuitively, $\mathcal{T}_{2}$ and $\mathcal{T}_{3}$ each contain more information about the $\Sigma$-concept names $B_{1}, B_{2}$ and $B_{4}$ than $\mathcal{T}_{1}$. We therefore propose a new measure based on the notions of minimal projection module and query module for different query languages.

Definition 8 (Projection Rate). Let $Q \in\{c, i, q\}$ and let $\mathcal{M}_{\rho}^{Q}$ range over minimal projection modules under $\rho=\left\langle\mathcal{T}_{1}, \Sigma, \mathcal{T}_{2}\right\rangle$ and the query type $Q$. Additionally, let $M_{\Sigma}^{Q}$ range over minimal modules of $\mathcal{T}_{2}$ for the query type $Q$. The projection rate $P^{Q}$ of $\mathcal{T}_{1}$ over $\mathcal{T}_{2}$ is defined as:

$$
P^{Q}=\frac{\left|\bigcup \mathcal{M}_{\rho}^{Q}\right|}{\left|\bigcup M_{\Sigma}^{Q}\right|}
$$

Note that $p \leq 1$ always holds by Lemma 3 . Intuitively, the lower the projection rate, the more $\Sigma$-knowledge is contained in $\mathcal{T}_{2}$ compared with $\mathcal{T}_{1}$.

Example 5 (Ex. 4 contd.). Considering when $Q=c$, we have that $\mathcal{M}_{\rho}^{c}=\mathcal{M}_{\rho^{\prime}}^{c}=\left\{\alpha_{1}\right\}$ under $\rho=\left\langle\mathcal{T}_{1}, \Sigma, \mathcal{T}_{2}\right\rangle$ and $\rho^{\prime}=\left\langle\mathcal{T}_{1}, \Sigma, \mathcal{T}_{3}\right\rangle$. The minimal subsumption query module of $\mathcal{T}_{2}$ w.r.t. $\Sigma$ is $\left\{\alpha_{1}, \alpha_{2}\right\}$. But there exists two minimal subsumption query module of $\mathcal{T}_{3}$ w.r.t. $\Sigma$, which are $\left\{\alpha_{1}, \alpha_{2}, \alpha_{3}\right\}$ and $\left\{\alpha_{1}, \alpha_{2}, \alpha_{4}\right\}$. So the union of minimal subsumption query module of $\mathcal{T}_{3}$ w.r.t. $\Sigma$ is $\mathcal{T}_{3}$ that contains four axioms. Therefore, the projection rate $P^{c}$ of $\mathcal{T}_{1}$ over $\mathcal{T}_{2}$ is $P^{c}=1 / 2$ and the projection rate $P^{c}$ of $\mathcal{T}_{1}$ over $\mathcal{T}_{3}$ is $P^{c}=1 / 4$. So $\mathcal{T}_{3}$ contains more $\Sigma$-knowledge compared with $\mathcal{T}_{2}$ as $1 / 4<1 / 2$. 


\section{Conclusion}

We proposed a novel module notion called projection module that entails the queries that follow from a reference ontology. We presented an algorithm for computing all minimal projection modules of acyclic $\mathcal{E} \mathcal{L} \mathcal{H}^{r}$-terminologies and two applications of minimal projection modules. We expect that the algorithms can be extended to deal with cyclic terminologies and possibly general $\mathcal{E} \mathcal{L H}^{r}$ TBoxes, and to yield a ranking between different projection modules, e.g., via weighted signatures.

\section{References}

1. Alsubait, T., Parsia, B., Sattler, U.: Measuring similarity in ontologies: A new family of measures. In: Proceedings of EKAW'14: the 19th International Conference on Knowledge Engineering and Knowledge Management. pp. 13-25 (2014)

2. Arif, M.F., Mencía, C., Ignatiev, A., Manthey, N., Peñaloza, R., Marques-Silva, J.: BEACON: An efficient SAT-based tool for debugging $\mathrm{EL}^{+}$-ontologies. In: Proceedings of SAT'16: the 19th International Conference on the Theory and Applications of Satisfiability Testing. pp. 521-530 (2016)

3. Baader, F., Calvanese, D., McGuinness, D.L., Nardi, D., Patel-Schneider, P.F. (eds.): The description logic handbook: theory, implementation, and applications. Cambridge University Press, 2 edn. (June 2010)

4. Baader, F., Horrocks, I., Lutz, C., Sattler, U.: An Introduction to Description Logic. Cambridge University Press (2017)

5. Baader, F., Peñaloza, R., Suntisrivaraporn, B.: Pinpointing in the description logic EL. In: Proceedings of DL'07: the 20th International Workshop on Description Logics (2007)

6. Botoeva, E., Konev, B., Lutz, C., Ryzhikov, V., Wolter, F., Zakharyaschev, M.: Inseparability and conservative extensions of description logic ontologies: A survey. In: Reasoning Web Summer School 2016, LNCS, vol. 9885, pp. 27-89. Springer International Publishing (2017)

7. Botoeva, E., Kontchakov, R., Ryzhikov, V., Wolter, F., Zakharyaschev, M.: Games for query inseparability of description logic knowledge bases. Artificial Intelligence $234,78-119(2016)$

8. Botoeva, E., Lutz, C., Ryzhikov, V., Wolter, F., Zakharyaschev, M.: Query-based entailment and inseparability for alc ontologies. In: Proceedings of IJCAI'16: the 25th International Joint Conference on Artificial Intelligence. pp. 1001-1007. AAAI Press (2016)

9. Chen, J., Ludwig, M., Ma, Y., Walther, D.: Zooming in on ontologies: Minimal modules and best excerpts. In: Proceedings Part I of ISWC'17: the 16th International Semantic Web Conference. pp. 173-189 (2017)

10. Chen, J., Ludwig, M., Walther, D.: Computing minimal subsumption modules of ontologies. In: Proceedings of GCAI'18: the 4th Global Conference on Artificial Intelligence. pp. 41-53 (2018)

11. Domingue, J., Anutariya, C. (eds.): Proceedings of ASWC'08: the 3rd Asian Semantic Web Conference on The Semantic Web, Lecture Notes in Computer Science, vol. 5367. Springer (2008) 
12. Ecke, A., Ludwig, M., Walther, D.: The concept difference for $\mathcal{E} \mathcal{L}$-terminologies using hypergraphs. In: Proceedings of DChanges'13: the International workshop on (Document) Changes: modeling, detection, storage and visualization (2013)

13. Euzenat, J., Shvaiko, P.: Ontology Matching, Second Edition. Springer (2013)

14. Grau, B.C., Horrocks, I., Kazakov, Y., Sattler, U.: Modular reuse of ontologies: Theory and practice. Journal of Artificial Intelligence Research 31(1), 273-318 (2008)

15. Jung, J.C., Lutz, C., Martel, M., Schneider, T.: Query conservative extensions in horn description logics with inverse roles. In: Proceedings of IJCAI'17: the 26th International Joint Conference on Artificial Intelligence. pp. 1116-1122. AAAI Press (2017)

16. Kalyanpur, A., Parsia, B., Sirin, E., Hendler, J.A.: Debugging unsatisfiable classes in OWL ontologies. Journal of Web Semantics 3(4), 268-293 (2005)

17. Kazakov, Y., Skocovsky, P.: Enumerating justifications using resolution. In: Proceedings of DL'17: the 30th International Workshop on Description Logics (2017)

18. Konev, B., Kontchakov, R., Ludwig, M., Schneider, T., Wolter, F., Zakharyaschev, M.: Conjunctive query inseparability of OWL 2 QL TBoxes. In: Proceedings of AAAI'11: the 25th Conference on Artificial Intelligence. AAAI Press (2011)

19. Konev, B., Ludwig, M., Walther, D., Wolter, F.: The logical difference for the lightweight description logic EL. Journal of Artificial Intelligence Research 44, 633-708 (2012)

20. Konev, B., Lutz, C., Walther, D., Wolter, F.: Semantic modularity and module extraction in description logics. In: Proceedings of ECAI'08: the 18th European Conference on Artificial Intelligence. pp. 55-59. IOS Press (2008)

21. Konev, B., Lutz, C., Walther, D., Wolter, F.: Formal Properties of Modularisation, pp. 25-66. Springer Berlin Heidelberg, Berlin, Heidelberg (2009)

22. Konev, B., Lutz, C., Walther, D., Wolter, F.: Model-theoretic inseparability and modularity of description logic ontologies. Artificial Intelligence 203, 66-103 (2013)

23. Konev, B., Walther, D., Wolter, F.: The logical difference problem for description logic terminologies. In: Proceedings of IJCAR'08. pp. 259-274 (2008)

24. Konev, B., Walther, D., Wolter, F.: The logical difference problem for description logic terminologies. In: Proceedings of IJCAR'08. pp. 259-274 (2008)

25. Kontchakov, R., Wolter, F., Zakharyaschev, M.: Logic-based ontology comparison and module extraction, with an application to DL-Lite. Artificial Intelligence 174(15), 1093-1141 (2010)

26. Lehmann, K., Turhan, A.: A framework for semantic-based similarity measures for ELH-concepts. In: Proceedings of JELIA'12: the 13th European Conference Logics in Artificial Intelligence. Lecture Notes in Computer Science, vol. 7519, pp. 307-319. Springer (2012)

27. Ludwig, M., Walther, D.: The logical difference for ELHr-terminologies using hypergraphs. In: Proceedings of ECAI'14: the 21st European Conference on Artificial Intelligence. pp. 555-560 (2014)

28. Romero, A.A., Kaminski, M., Grau, B.C., Horrocks, I.: Module extraction in expressive ontology languages via datalog reasoning. Journal of Artificial Intelligence Research 55, 499-564 (2016)

29. Sattler, U., Schneider, T., Zakharyaschev, M.: Which kind of module should I extract? In: Proceedings of DL'09. CEUR Workshop Proceedings, vol. 477. CEURWS.org (2009)

30. Zhou, Z., Qi, G., Suntisrivaraporn, B.: A new method of finding all justifications in OWL 2 EL. In: Proceedings of WI'13: IEEE/WIC/ACM International Conferences on Web Intelligence. pp. 213-220 (2013) 